\begin{tabular}{||l|l|}
\hline Citation & $\begin{array}{l}\text { Piet Callemeyn, Michiel Steyaert } \\
\text { Monolithic Integration of a Class DE Inverter for On-Chip Resonant DC- } \\
\text { DC converters } \\
\text { European Solid-State Circuits Conference } 2012 \text { (pp.325 - 328) }\end{array}$ \\
\hline Archived version & $\begin{array}{l}\text { Author manuscript: the content is identical to the content of the published } \\
\text { paper, but without the final typesetting by the publisher }\end{array}$ \\
\hline Published version & http://dx.doi.org/10.1109/ESSCIRC.2012.6341320 \\
\hline Journal homepage & http://www.ims-bordeaux.fr/ESSCIRC2012 \\
\hline Author contact & $\begin{array}{l}\text { piet.callemeyn@esat.kuleuven.be } \\
+32(0) 16321086\end{array}$ \\
\hline
\end{tabular}

(article begins on next page) 


\title{
Monolithic Integration of a Class DE Inverter for On-Chip Resonant DC-DC Converters
}

\author{
Piet Callemeyn and Michiel Steyaert \\ ESAT-MICAS, KU Leuven, \\ Kasteelpark Arenberg 10, \\ B-3001 Leuven, Belgium \\ Email: piet.callemeyn@esat.kuleuven.be
}

\begin{abstract}
A fully-integrated series resonant class DE inverter is realized in a $130 \mathrm{~nm} 1.2 \mathrm{~V}$ CMOS technology with an on-chip spiral inductor and an integrated MIMcap. It is used as the first stage in fully-integrated class DE DC-DC resonant converters. The inherent soft switching yields high conversion efficiency at high switching frequencies. Low switching peakvoltages are present in the circuit, alleviating the need for onchip high-voltage techniques. The use of on-chip passives reduces the bill of materials (BOM) considerably. The maximum output power of the series resonant class $\mathrm{DE}$ inverter is $11.6 \mathrm{~mW}$. The maximum power efficiency is $65.2 \%$. The measurement results confirm and improve previous simulations.
\end{abstract}

\section{INTRODUCTION}

One of the major techniques to reduce cost, size and power consumption in state-of-the-art applications is the monolithic integration of electronic systems. This trend was first observed in the successful integration of RF mixed-signal building blocks [1]. A following step in this integration paradigm is the integration of another building block: the power supply. Recent research efforts cleared the path to develop fully-integrated DC-DC converters in standard CMOS [2].

When all the building blocks are integrated on a single die, multiple supply voltages are required. For example, the digital circuitry will need a lower supply voltage than the on-chip power amplifier which must deliver enough power into the communication medium. Therefore, multiple voltage domains need to be created to accommodate for all different supply voltages.

Because of the continuing miniaturization, battery-powered portable wireless applications result. The battery voltage is usually too high for the deep-submicron technologies used. Therefore, the supply voltages need to be decreased. This is typically done using low efficient linear regulators. To reduce the powerlosses, on-chip switching power supplies can be used, increasing the efficiency and battery lifetime. An interesting application is the supply for digital memories, requiring low supply voltages to reduce the dynamic losses.

Among the switching power supplies, the resonant switching DC-DC converters achieve high efficiency using a resonant circuit and high frequency inverters. Examples are class E inverters [3] and class DE inverters [4] achieving high efficiency at high switching frequencies due to the inherent decreased switching losses in these topologies. These reduced switching losses are inherent for resonant circuits as will be explained

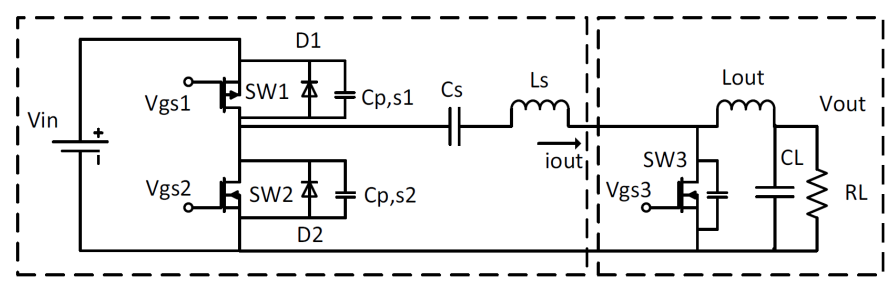

(a) Class DE DC-DC converter with series resonant class DE inverter (left) and class E synchronous rectifier (right).

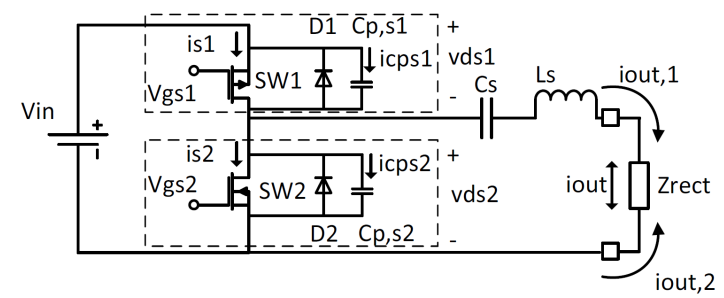

(b) Equivalent circuit used for the design of the series resonant class DE inverter.

Fig. 1. Class DE DC-DC converter circuit and equivalent schematic.

later. In a class $\mathrm{E}$ inverter, high peak switching voltages are present, up to four times the supply voltage. However, the breakdown voltage for transistors in deep-submicron technologies is low, requiring measures to prevent degradation or breakdown of the switches. For a class DE inverter on the other hand, the peak switch voltage will be limited to the supply voltage. It is thus more suitable for integration in deepsubmicron technologies.

This paper presents a resonant class DE DC-DC converter, using a fully-integrated series resonant class DE inverter as a first stage. The architecture and operation principles of the converter are described in section II. The implementation of the most important components of the series resonant class DE inverter is discussed in Section III. Section IV describes the measurement results. Finally, conclusions are drawn in section V.

\section{ARCHITECTURE}

\section{A. Class DE DC-DC converter}

Figure 1(a) illustrates the schematic of a resonant class DE DC-DC converter with ideal components.

It consists of a series resonant class DE inverter and a synchronized class E rectifier. The inverter is loaded with the 
input impedance $Z_{\text {rect }}$ of the rectifier. This is shown in Figure 1(b) as an equivalent circuit which is useful for analysis and is used for the design of the series resonant class DE inverter.

A resonant power converter has some advantages over switching PWM converters. These PWM converters will suffer from switching losses and low efficiency at high frequencies [5]. The chief advantage of resonant converters is their reduced switching loss. This is achieved via mechanisms known as zero-current switching (ZCS) and zero-voltage switching (ZVS). The turn-on and turn-off transitions of the various converter switches can occur at zero crossings of the resonant converter quasi-sinusoidal waveforms. This will reduce switching losses, meaning that resonant converters can be operated at higher switching frequencies than PWM converters. Zerovoltage switching can also eliminate some of the sources of converter-generated electromagnetic interference.

The series resonant class $\mathrm{DE}$ inverter generates an $\mathrm{AC}$ voltage which is fed into a series resonant LC-tank, formed by $L_{s}$ and $C_{s}$. Thereafter, it is fed into the synchronized class $\mathrm{E}$ rectifier. The DC output voltage of the rectifier is filtered by output capacitor $C_{L}$ and is also the output voltage $V_{\text {out }}$ of the converter. The voltage conversion ratio $k\left(f_{S W}\right)$ of the converter is calculated by means of the transfer function $\left\|H\left(f_{S W}\right)\right\|$ between the input and the output. This is further elaborated in [6], but out of scope to be discussed in this paper.

\section{$B$. Series resonant class DE inverter}

Figure 1(b) illustrates the schematic of a series resonant class DE inverter with ideal components. It consists of two MOS transistors $S W 1$ and $S W 2$, a MIM-capacitor $C_{s}$, an inductor $L_{s}$ and a resistive output load $Z_{\text {rect }}$. The capacitor, inductor and resistor form the series resonant tank. The capacitors $C_{p, s 1}$ and $C_{p, s 2}$ are the parasitic output capacitances of the MOS transistors. Diodes $D 1$ and $D 2$ are the intrinsic body-drain pn-junction diodes. These will be used as antiparallel diodes. The switches turn on and off periodically. This is controlled by a non-overlapping clock: there is a dead-time between the switch on-times to prevent short-circuit currents flowing through both transistors. The operation is described as follows. During the first switching stage $S W 1$ is closed and $S W 2$ is open. The current $i_{\text {out }, 1}$ through the inductor and capacitor now starts to flow. During the second stage $S W 1$ is open and $S W 2$ is closed. The current $i_{\text {out }, 2}$ due to the stored magnetic and electric energy in the resonant tank now flows in the opposite direction of $i_{\text {out }, 1}$. Over one complete period, the current $i_{\text {out }}$ through this resonant tank is nearly a sine wave. The output load $Z_{\text {rect }}$ sees this sinusoidal current that changes direction every clock cycle, this yields an AC voltage across the load resistor. This principle of operation is also explained by the waveforms, sketched in Figure 2 .

1) Inherent reduced switching losses: An interesting feature of this topology is the inherent reduced switching loss compared to a classic pulse-width modulated (PWM) switch-mode power supply. Figure 2 shows the waveforms for the operation at, below and above the resonant frequency of the series resonant converter. For operation at the resonant frequency,

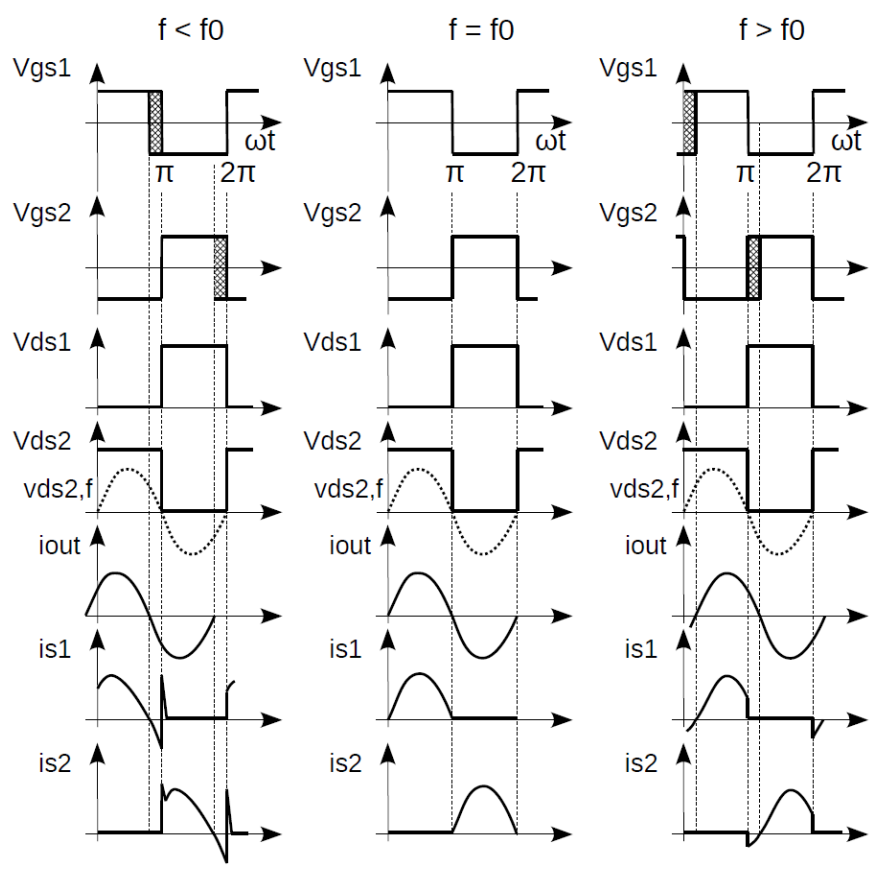

Fig. 2. Waveforms for operation at $f<f_{0}, f=f_{0}$ and $f>f_{0}$.

$f=f_{0}$, the transistors turn on and off at zero current, resulting in low switching losses and high efficiency. In many cases, the operating frequency $f$ is not equal to the resonant frequency $f_{0}$ since the output voltage and power can be controlled by varying the operating frequency $f$. Each transistor should be turned off for $f<f_{0}$ and turned on for $f>f_{0}$ during the time interval when the switch current is negative. During this time, the current can flow through the anti-parallel diode. To prevent short-circuit currents, a non-overlapping clock with sufficient dead-time must be used.

When the inverter is operated below resonance, zero-current switching (ZCS) will occur. The series resonant circuit will represent a capacitive load [7], this means that the current $i$ through the resonant tank will lead the fundamental component $v_{d s 2, f}$ of the voltage $v_{d s 2}$. In this case, the transistor current goes to zero before the transistor is turned off. The circuit inherently causes the turn-off transition to be lossless. However, when the transistor is turned on, its parasitic output capacitance is discharged through its on-resistance, causing a switching loss.

When the inverter is operated above resonance, zero-voltage switching (ZVS) will occur. The series resonant circuit now represents an inductive load. The circuit naturally causes the transistor voltage to become zero before the transistor is turned on. The current $i$ lags $v_{d s 2, f}$. The turn-on transition is now lossless. Both the switch voltage and current waveforms overlap during turn-off, causing a switching loss.

2) Exploiting the parasitic output capacitances: To assist the turn-off process above resonance, small shunt capacitors can be introduced in parallel with the transistors. The transistors used in this circuit are sufficiently large to use the output capacitances $C_{p, s 1}$ and $C_{p, s 2}$ as shunt capacitors. These shunt capacitors eliminate the turn-off switching loss 


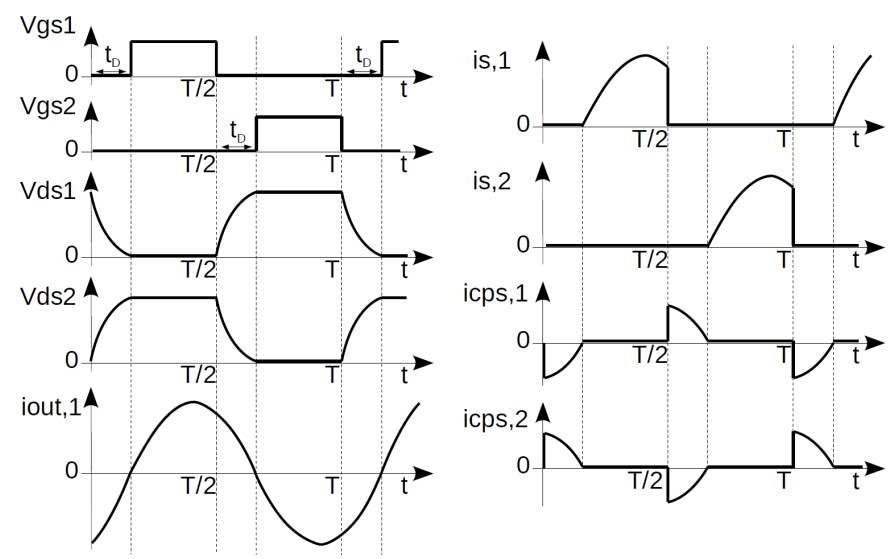

Fig. 3. Waveforms for the series resonant class DE inverter.

during operation above the resonant frequency. This principle is depicted in Figure 3. There is again a dead time in the gate-to-source voltages, during which both transistors are off. During the dead time, shunt capacitors become part of the resonant circuit. One shunt capacitor is charged and the other is discharged during the dead time. These capacitors introduce commutation intervals at transistor turn-off. When $S W 1$ is turned off, the tank current flows through capacitance $C_{p, s 1}$ instead of $S W 1$ itself and the voltage across $S W 1$ and $C_{p, s 1}$ increases. If the turn-off time is sufficiently fast, the transistor is turned off before the drain voltage rises too much above zero. A negligible switching loss is now incurred. This series resonant converter achieves the class E switching conditions [3], which means zero-voltage switching and zero-voltage slope switching. This topology enables higher efficiency since it eliminates switching power losses during the transition time of the switches.

\section{Class E synchronous rectifier}

The right part of the resonant DC-DC converter in Figure 1(a) is the class $\mathrm{E}$ synchronous rectifier. This is a resonant rectifier [8]: a sinusoidal input current $i_{\text {out }}$ will be translated into a DC output current. This current will be limited by the output inductor $L_{\text {out }}$. Instead of a diode in a typical class E rectifier, a synchronized switch $S W 3$ is used. This circuit can operate efficiently at high frequencies because the switch turns on and off at zero voltage (low dv/dt) and turns off at zero current (low $\mathrm{di} / \mathrm{dt}$ ). This reduces the switching losses significantly. High efficiencies are reported for this type of monolithic synchronous rectifiers [9].

\section{IMPLEMENTATION}

The series resonant class DE inverter is implemented as a part of a resonant class DE DC-DC converter. The implementation of the synchronous class $\mathrm{E}$ rectifier is omitted here. In this paper, the focus is on the efficient design of the series resonant class DE inverter as a fully-integrated building block to achieve high frequent and highly efficient resonant DC-DC converters. The design and layout of the different components in the series resonant class DE inverter are discussed in this section. Since the class DE inverter is integrated on-chip

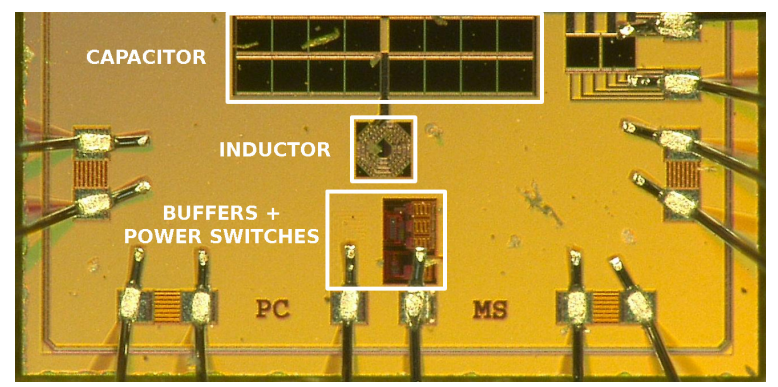

Fig. 4. Die photograph of the chip.

without rectifier, an external load resistor is needed to serve as an input impedance. This corresponds to the load $Z_{\text {rect }}$ in Figure 1(b).

The inductor is implemented as an octagonal metal track, hollow spiral inductor. A standard metal layer, a thick top metal layer of $2 \mu \mathrm{m}$, and an aluminium layer of $1.2 \mu \mathrm{m}$ are used to reduce the parasitic series resistance. It consists of 2 windings of $20 \mu \mathrm{m}$, resulting in a total on-chip inductance of $1.7 n H$. The load is connected off-chip using a bondwire, this introduces extra inductance. The total measured extracted inductance equals $5.1 \mathrm{nH}$. The series resistance at a frequency of $500 \mathrm{MHz}$ is $0.5 \Omega$, and is taken as an upper limit since the inverter is designed to be used around $100 \mathrm{MHz}$. The inductor area is $125 \mu \mathrm{m}$ by $125 \mu \mathrm{m}$. This can be seen in the middle of Figure 4.

The capacitor in the presented class DE inverter is implemented by means of a MIMCAP. In the used technology this yields a capacitance density of $2 \mathrm{fF} / \mu \mathrm{m}^{2}$. A total capacitance of $145.6 p F$ is realised. The electrical series resistance was estimated using the sheet resistances of the two metal layers, resulting in $0.8 \Omega$. The capacitor can be seen in the top part of Figure 4.

The power switches, $S W 1$ and $S W 2$ are implemented using a fingered layout. The width of the power switches is $1.8 \mathrm{~mm}$ and $0.75 \mathrm{~mm}$ respectively. This width yields an optimal tradeoff between losses in the buffers driving the switches and the conduction losses. The switches are driven by two buffer trains. The buffers apply the non-overlapping clock to the pMOS $(S W 1)$ and nMOS $(S W 2)$ transistor. These buffers and power switches are located in the bottom part of Figure 4.

\section{MeAsurements}

A series resonant class DE inverter is implemented in a $130 \mathrm{~nm} 1.2 \mathrm{~V}$ CMOS technology. It measures $1.5 \mathrm{~mm}$ x $0.75 \mathrm{~mm}$. Figure 4 shows a die photograph. Figure 5 shows the efficiency of the inverter as a function of the output power at varying resistive loads. The maximum efficiency is $65.2 \%$ at a load of $6.9 \mathrm{~mW}$ with an output peak amplitude of $585 \mathrm{mV}$ at a switching frequency of $100 \mathrm{MHz}$. A maximal output power of $11.6 \mathrm{~mW}$ is achieved at an efficiency of $47 \%$ with an output peak amplitude of $312 \mathrm{mV}$. Figure 6 shows the efficiency of the inverter in function of the switching frequency for a load of $25 \Omega$. An efficiency of $65.2 \%$ is achieved. At 


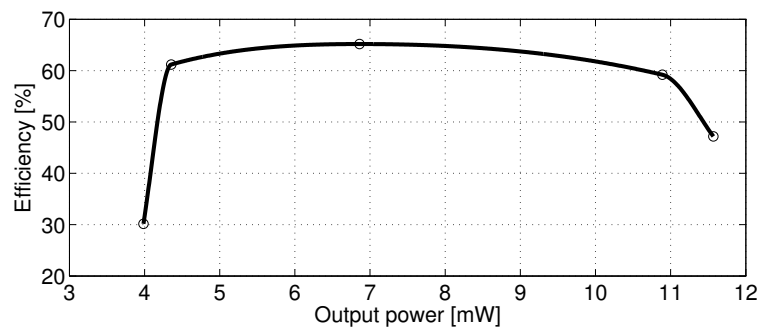

Fig. 5. The efficiency of the series resonant class DE inverter for varying loads.

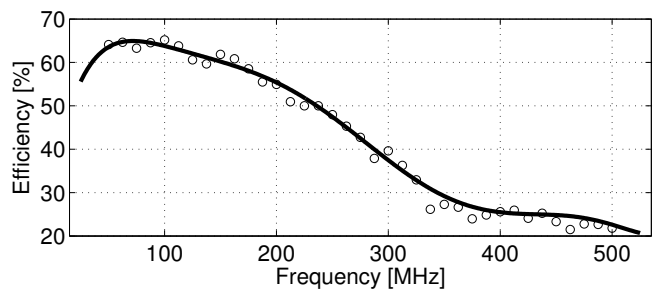

Fig. 6. The efficiency at increasing frequency for a $25 \Omega$ load.

a higher switching frequency, the efficiency decreases. It is paramount to control the switching frequency as to achieve the highest efficiency. This point is shifted depending on the output load. For this integrated circuit, the efficient switching frequency will be around $100 \mathrm{MHz}$, depending on the load used. In Figure 7 the output voltage sine wave at $100 \mathrm{MHz}$ is presented for a load of $25 \Omega$. The peak amplitude of the output waveforms is given in Figure 8 in function of the switching frequency. There is a peak around $100 \mathrm{MHz}$. It is seen that the amplitude of the output waveform can be controlled by the switching frequency. This range of amplitudes is interesting for the on-chip supply for digital memories. Table I summarizes the measurement results and makes a comparison with existing simulations of an integrated series resonant class DE inverter [4]. The measurements of the discussed monolithic series resonant class DE inverter confirm these simulation results. Moreover, the performance is increased compared to the simulated results.

\section{CONCLUSION}

A fully-integrated series resonant class DE inverter is realized in a $130 \mathrm{~nm} 1.2 \mathrm{~V}$ CMOS technology with an onchip spiral inductor and an integrated MIMcap. This circuit

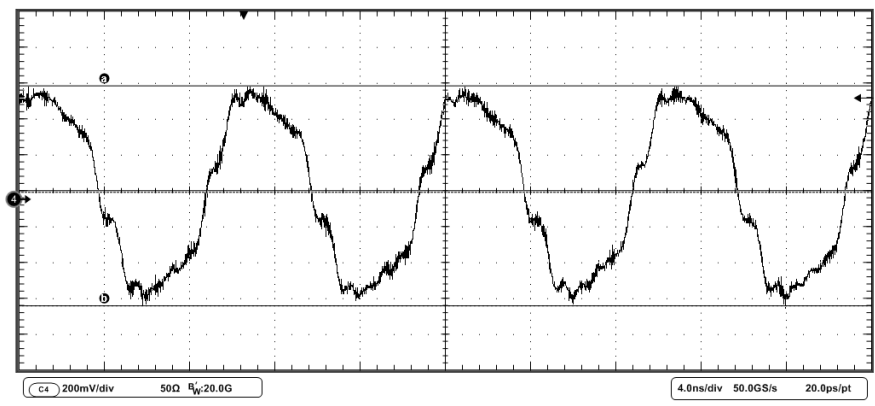

Fig. 7. Measured output voltage waveform at $100 \mathrm{MHz}$ for a $25 \Omega$ load.

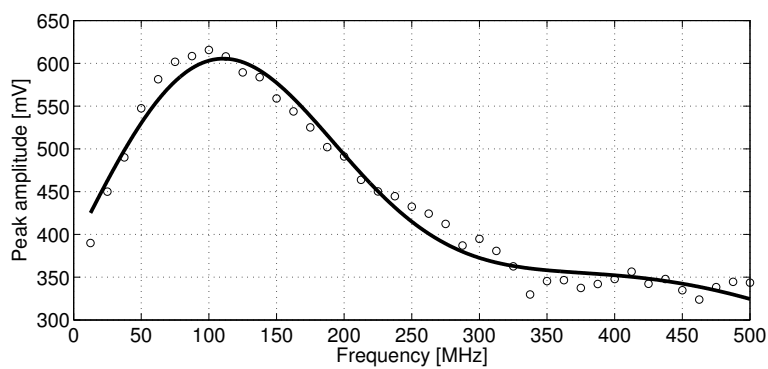

Fig. 8. Peak output amplitude as a function of switching frequency. TABLE I

SUMMARY OF THE MEASUREMENT RESULTS

\begin{tabular}{|c|c|c|}
\hline Reference & {$[4]$} & this work \\
\hline Process $(\mu \mathrm{m})$ & $1.2 \mu \mathrm{m}$ & $0.13 \mu \mathrm{m}$ \\
Input voltage & $2 \mathrm{~V}$ & $1.2 \mathrm{~V}$ \\
Maximum output peak amplitude & $320 \mathrm{mV}$ & $600 \mathrm{mV}$ \\
Maximum efficiency & $48 \%$ & $65.2 \%$ \\
Power at maximum efficiency & $6.4 \mathrm{~mW}$ & $6.9 \mathrm{~mW}$ \\
Switching frequency & $500 \mathrm{MHz}$ & $100 \mathrm{MHz}$ \\
Maximum output power @ efficiency & not available & $11.6 \mathrm{~mW} @ 47 \%$ \\
Notes & simulation only & fully-integrated \\
\hline
\end{tabular}

is intended for a resonant class DE DC-DC converter. The inherent soft switching yields high conversion efficiency at high switching frequencies. The use of on-chip passives reduces the bill of materials considerably. A maximum output power of $11.6 \mathrm{~mW}$ is achieved and a maximum efficiency of $65.2 \%$ without the need for external components. The output voltage peak amplitude lies around $600 \mathrm{mV}$ at a switching frequency of $100 \mathrm{MHz}$. These results confirm and improve previous simulations.

\section{REFERENCES}

[1] M. Steyaert and P. Vancorenland, "Cmos: a paradigm for low power wireless?" in Design Automation Conference, 2002. Proceedings. 39th, 2002, pp. $836-841$.

[2] M. Steyaert, T. Van Breussegem, H. Meyvaert, P. Callemeyn, and M. Wens, "Dc-dc converters: From discrete towards fully integrated cmos," in ESSCIRC, 2011 Proceedings of the, sept. 2011, pp. $42-49$.

[3] N. Sokal, "Class e high-efficiency switching-mode tuned power amplifier with only one inductor and one capacitor in load network-approximate analysis," JSSC, vol. 16, no. 4, pp. 380 - 384, aug 1981.

[4] T. Suetsugu and M. Kazimierczuk, "Integration of class de inverter for on-chip dc-dc power supplies," in Circuits and Systems, 2006. ISCAS 2006. Proceedings. 2006 IEEE International Symposium on, may 2006, p. 4 pp.

[5] B. Sahu and G. Rincon-Mora, "An accurate, low-voltage, cmos switching power supply with adaptive on-time pulse-frequency modulation (pfm) control," Circuits and Systems I: Regular Papers, IEEE Transactions on, vol. 54, no. 2, pp. $312-321$, feb. 2007.

[6] R. Erickson and D. Maksimovic, Fundamentals of power electronics. Springer, 2004.

[7] M. Kazimierczuk and D. Czarkowski, Resonant power converters. John Wiley \& Sons Inc, 2010.

[8] M. Fujii, T. Suetugu, H. Koizumi, K. Shinoda, and S. Mori, "Resonant $\mathrm{dc} / \mathrm{dc}$ converter with class e inverter and class e synchronous rectifier using thinned-out method," in ISCAS, 1995, pp. 1119 -1122 vol.2.

[9] C. Y. Lim, Y. Liang, G. Samudra, and N. Balasubramanian, "A smartpower synchronous rectifier by cmos process," Power Electronics, IEEE Transactions on, vol. 25, no. 9, pp. 2469 -2477, sept. 2010. 